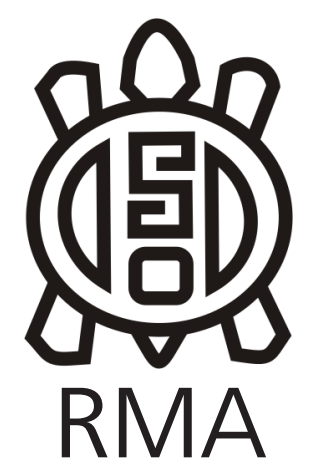

Dossier

\title{
Útiles en acción: instrumentalización e instrumentación en el análisis tecno-funcional de conjuntos líticos tardíos de la Costa Central de Perú
}

Tools in action: instrumentalisation and instrumentation in the technofunctional analysis of late lithic assemblages from the Central Coast of Peru

Antonio Pérez Balarezo*, Pilar Babot** y Lucénida Carrión Sotelo***

*Universidad Nacional de Trujillo, Perú y Université de Rennes, Francia. E-mail: antonioperezbalarezo@hotmail.com

Actual filiación institucional: Université Paris Ouest - Nanterre La Défense, CNRS - UMR 7041 ArScAn-Maison de I'Archéologie et de l'Ethnologie, 200 Avenue de la République, 92001 Nanterre Cedex. **Instituto Superior de Estudios Sociales, CONICET; Instituto de Arqueología y Museo, Universidad Nacional de Tucumán. San Martín 1545, CP 4.000 San Miguel de Tucumán, Argentina. E-mail: pilarbabot@yahoo.com *** División de Arqueología, Parque de las Leyendas "Felipe Benavides Barreda", Las Leyendas 580, 15008 Lima, Perú. E-mail: lucenida.carrion@leyendas.gob.pe

\begin{abstract}
Resumen
El presente trabajo analiza la viabilidad metodológica de la aplicación de categorías propias de la ergonomía francesa en el análisis tecno-funcional de nueve conjuntos líticos del Complejo Arqueológico Maranga (Costa Central peruana), adscritos a períodos tardíos (ca. 1.800-400 AP). Desde un enfoque que considera a los útiles siempre en acción, se aplican las categorías de instrumentalización e instrumentación a fin de evaluar su pertinencia para la integración de las esferas de producción y funcionamiento. De esta manera, se diseñó un programa metodológico capaz de integrar diferentes técnicas especializadas (petrografía, Difracción de Rayos $X$, tafonomía, tecnología y de microvestigios vegetales) a fin de identificar los esquemas operatorios de producción y funcionamiento recurrentes en cada conjunto lítico. Los resultados obtenidos a partir de cada técnica se evalúan en función del grado de coherencia teórico-metodológica que expresan entre sí, y su relevancia para el estudio sincrónico y diacrónico de la variabilidad instrumental en la Costa Central peruana.
\end{abstract}

Palabras clave: Instrumentalización; Instrumentación; Tecnología lítica; Períodos tardíos; Costa Central peruana.

\begin{abstract}
This paper analyzes the methodological viability of the application of concepts from French ergonomics in the techno-functional analysis of nine lithic assemblages of the Maranga Archaeological Complex (Central Coast of Peru), assigned to late periods (ca. 1.800-400 BP). From an approach that considers that tools exist only in action, the instrumentalization and instrumentation categories are applied in order to evaluate their relevance for the integration of the spheres of production and functioning. In this way, a methodological program was designed to integrate different specialized techniques (petrography, X-ray diffraction, taphonomy, technology and plant microremains) in order to identify the operational patterns of recurrent production and use in each lithic assemblage. The results obtained from each technique are evaluated according to the degree of theoretical-methodological coherence they express, and their relevance for the synchronic and diachronic study of instrumental variability in the Central Coast of Peru.
\end{abstract}

Keywords: Instrumentalization; Instrumentation; Lithic technology; Late periods; Central Coast of Peru.

\section{Introducción}

La arqueología de la Costa Central peruana constituye un amplio conjunto de estudios realizados desde la primera mitad del siglo XIX. Esta profundidad temporal no necesariamente ha repercutido en la diversificación de la investigación arqueológica en esta parte de la región andina, concentrada principalmente en el estudio de la arquitectura monumental, estilos cerámicos y contextos funerarios propios de sociedades complejas (v. g. Canziani, 2009; Díaz \& Vallejo, 2004; Kaulicke, 2000; Makowski, 2002; Mauricio, Prieto, \& Pardo, 2014).

Como resultado, el panorama arqueológico de esta región es todavía fragmentario. Después de la influencia Chavín en la Costa Central, las expresiones culturales 
de los períodos tardíos demuestran la consecución de una vida netamente urbana asociada a organizaciones políticas estatales multivalle, hecho que se inició entre el fin del Período Intermedio Temprano (ca. 2.200-1.450 AP) y el inicio del Horizonte Medio (ca. 1450-1050 AP) (Makowski, 2001; Marcone, 2015). Así, desde el Período Intermedio Temprano al Horizonte Tardío (ca. 470-400 $A P)$, esta región estuvo ocupada principalmente por las denominadas culturas Lima, Wari, Ichma e Inca. Las sociedades Lima e Ichma construyeron importantes centros urbanos con edificios piramidales de carácter monumental, siendo Maranga el principal de ellos, desde la fase cerámica Lima Medio (ca. 2000-1450 AP) (Narváez, 2013). Por su parte, se conoce que la dominación inca tuvo un impacto reducido en las esferas de producción y consumo tecnológico y estilístico, sea ya en la producción alfarera o en la arquitectónica; es decir los artesanos mantuvieron sus tradicionales formas de producción.

En este contexto, el material lítico correspondiente a períodos tardíos generalmente no ha sido considerado como fuente de conocimiento sobre la actividad artesanal pasada. Uno de los escasos trabajos de investigación describe el material de forma breve, relegándolos a las etiquetas de artefactos poco elaborados y no estructurados (Taschini, 1968). El otro, inicia la investigación sistemática de la producción lítica en la región, considerando para su interpretación las estrategias tecnológicas expeditivas y conservadas (Silva, 2014).

Este trabajo, por consiguiente, se ha planteado continuar la investigación de la tecnología lítica de esta región, a partir de nueve sitios integrantes del Complejo Arqueológico Maranga, ubicados en el valle bajo del río Rímac, y ocupados entre ca. 2200 y 400 AP. El objetivo es caracterizar de forma global (sistemas de producción y funcionamiento) el sub-sistema técnico lítico presente en cada período considerado, a fin de estudiar el grado de variabilidad técnica y funcional presente en Maranga a través del tiempo.

\section{Útiles en acción: o sobre instrumentalización e instrumentación}

Generalmente, el enfoque de la chaîne opératoire (LeroiGourhan, 1964; Tixier, Inizan, \& Roche, 1980) se ha utilizado para el abordaje sincrónico de la producción lítica, capaz de reconstituir la temporalidad y espacialidad específicas de un modo de producción determinado, pero muchas veces dejando de lado el aspecto diacrónico del cambio técnico concerniente a los objetivos de producción. En busca de contribuir a este vacío, el enfoque tecno-funcional (Boëda, 2013), desde una perspectiva anclada en la filosofía de las técnicas y la ergonomía francesa (Rabardel, 1995), busca comprender las transformaciones que tienen lugar en las industrias líticas a través del tiempo, prestando atención a los objetivos de producción, es decir los útiles. Desde inicios de los años noventa (Boëda, 1991, 1997; Lepot, 1993), este enfoque parte de un análisis estructural del utillaje, integrando esferas de producción y confección, a fin de identificar las intenciones funcionales subyacentes, a través del estudio de las operaciones técnicas utilizadas (Boëda, 2013, p. 104).

El útil, entonces, se concibe como una entidad mixta compuesta por tres elementos: artefacto, esquemas de utilización y energía (Boëda, 2013, p. 46). Los primeros dos conciernen al estudio de los procesos de instrumentalización, mientras que el tercero a los procesos de instrumentación (Boëda, 2013, pp. 50-53; Rabardel, 1995, p. 111). Ambos procesos deben ser concebidos como un continuum de operaciones técnicas entre el artesano, el artefacto y la materia a trabajar. Los procesos de instrumentalización están compuestos por un conjunto de constreñimientos técnicos que pueden ser extrínsecos o intrínsecos a los útiles. Los primeros están referidos a las propiedades de la materia a trabajar, a la calidad, disponibilidad y accesibilidad de la materia prima, y a la memoria técnica que representa cada útil (Boëda, 2013, p. 44). Por su parte, los constreñimientos intrínsecos se refieren a la estructura interna de cada artefacto (volumen -límites tridimensionales de un determinado objeto, compuesto en el caso de un útil lítico por tres partes funcionales: transformativa, transmisora y prensil-y organización - relación de sinergia entre las partes funcionales del útil) y a sus reglas de funcionamiento (Boëda, 2013, p. 45). En otras palabras, la instrumentalización está referida a los esquemas de producción y confección que el artesano ejecuta sobre la materia prima. Complementariamente, los procesos de instrumentación se refieren al gesto ejecutado, la forma de prensión utilizada, la energía transmitida (movimiento) y el lugar seleccionado para el desarrollo de la acción técnica (Boëda, 2013, pp. 50-53). Nos referimos entonces a los esquemas de utilización que el artesano realiza con el artefacto producido, gracias a su experiencia. Un útil o instrumento, entonces, es una suerte de interfase entre ambos procesos. (Figura 1).

En base a estas concepciones, el abordaje de la génesis instrumental debe apoyarse en una lectura funcional de las operaciones técnicas utilizadas para modificar las materias primas y/o los soportes iniciales. Esto implica analizar las consecuencias funcionales de cada etapa de la cadena operativa, y estudiar la relación entre éstas y la materia a trabajar. Así, el enfoque tecno-funcional extiende y completa el alcance de la chaîne opératoire, que aborda el funcionamiento y uso instrumental a través de otros estudios fuera de la tecnología, como por ejemplo la traceología.

\section{Área de estudio}

Fueron seleccionados nueve sitios de cuatro períodos 


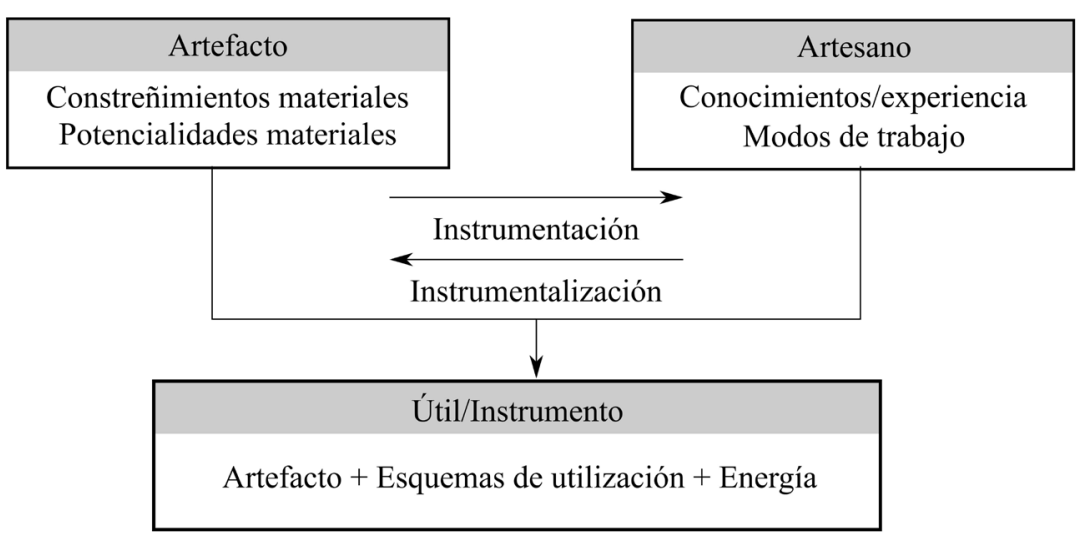

Figura 1: Génesis instrumental (Modificado de Boëda, 2013, fig. 4; Trouche, 2005, fig. 6.6).

Figure 1: Instrumental genesis (Modified from Boëda, 2013, fig. 4; Trouche, 2005, fig. 6.6).

fueron objeto de análisis tecnológico y petrográfico (Tabla 1), todos provenientes mayoritariamente de contextos secundarios (rellenos arquitectónicos), debido a la naturaleza arquitectónica de los sitios excavados,

arqueológicos diferentes, integrantes del Complejo Arqueológico Maranga (CAM). Gran parte de este complejo (54 sitios en total) se localiza en el territorio perteneciente a un zoológico moderno denominado Parque de las Leyendas-Felipe Benavides Barreda (PATPAL). Por este motivo, de aquí en adelante, el área de estudio será designada con las siglas CAMPATPAL.

Con un área total aproximada de $3 \mathrm{~km}^{2}$, el CAM-PATPAL se localiza en el valle bajo del río Rímac, margen izquierda, a 3.5 km del Océano Pacífico, en la Costa Central de Perú (Carrión \& Narváez, 2014, p. 33). Esta área está dominada por un relieve relativamente plano, variando entre los 48 y 68 msnm, y definida ecológicamente por la presencia de vegetación xerofítica dispersa (Figura 2).

En razón del grado de representatividad de la población artefactual y su variabilidad temporal, la selección de sitios comprendió: Huaca El Rosal, Huaca Middendorf, Huaca 33, Huaca 34, Huaca 43, Huaca San Miguel, Huaca 58B (sitios arqueológicos propiamente dichos), Murallas 46A y 55E (paisajes arqueológicos). La Figura 3 muestra la distribución espacial de los sitios al interior del CAM-PATPAL.

\section{Materiales y métodos}

Inicialmente, un total de 1108 artefactos tallados ampliamente caracterizados por masivas construcciones realizadas a través de una sucesión de volúmenes de relleno arquitectónico.

Al ser un trabajo inicial, no ha sido posible cubrir todos los elementos de la génesis instrumental. Por ello, en primer lugar, se parte del análisis petrográfico, a nivel macro y microscópico. Por un lado, la descripción macroscópica se realizó a través de una lupa binocular que permitió registrar: naturaleza de la roca, compacidad, color general o rango de colores de la muestra, textura, tamaño del grano, composición mineralógica, estructuras, evidencias de meteorización o alteración, presencia de xenolitos o fragmentos líticos, estimación cualitativa de densidad y presencia de magnestismo). Por otro lado, una vez preparadas las muestras de sección delgada se procedió a la descripción microscópica a través del microscopio de polarización Leica DM4 P. Para la descripción microscópica se consideraron las siguientes variables: naturaleza de la roca, textura, constituyentes (tanto los principales como los propios de la matriz, dependiendo del tipo de roca), alteraciones, microestructuras (tamaños, formas, abundancia relativa y materiales de relleno si estuvieran presentes), y fotomicrografía general de la muestra.

En segundo lugar, mediante de Difracción de Rayos $X$ (DRX), se procedió con la determinación precisa del tipo de rocas presentes en la colección. Particularmente,

Figura 2: Localización del Complejo Arqueológico Maranga (CAM), en la margen izquierda del valle bajo del río Rímac (Costa Central, Perú). (Modificado de: maps-for-free. com)

Figure 2: Location of Maranga Archaeological Complex (CAM), on the left bank of the lower Rimac River valley (Central Coast, Peru). (Modified from: maps-for-free.com)
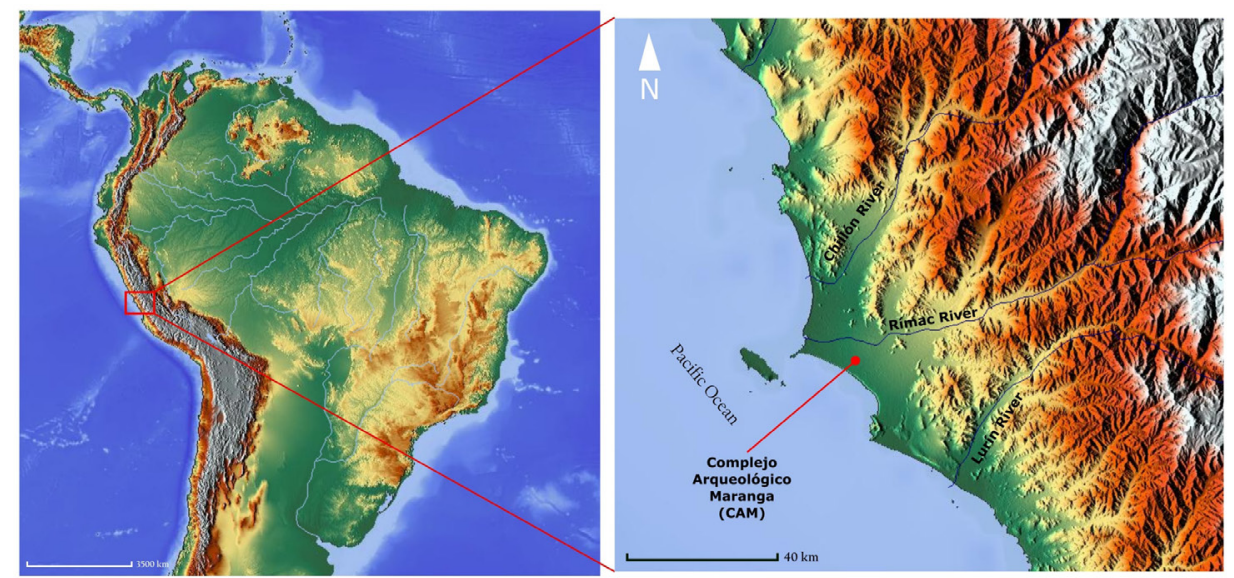


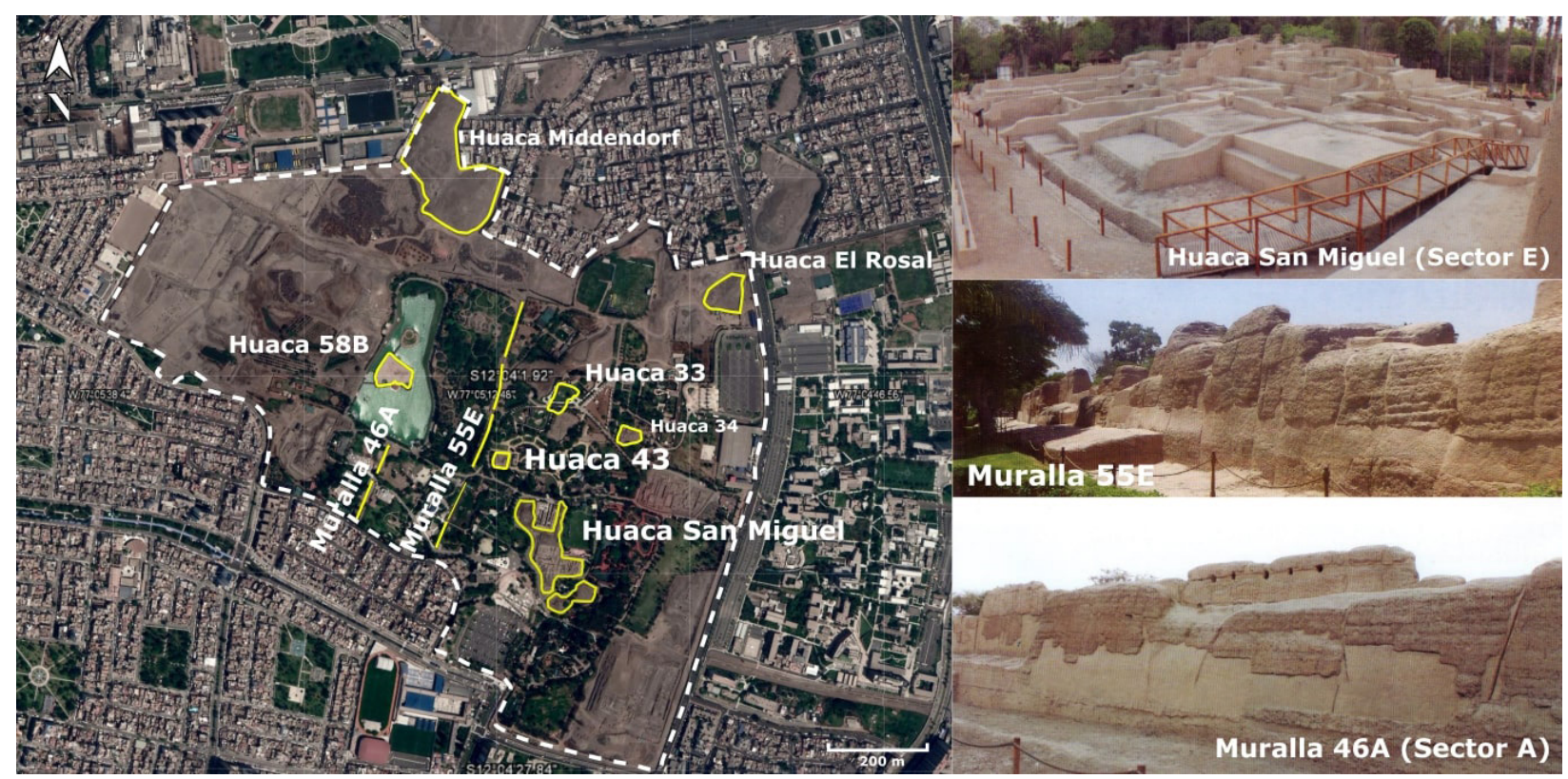

Figura 3: Izquierda: Plano de ubicación de los nueve sitios seleccionados. En interlineado blanco, perímetro del CAM en el PATPAL. En amarillo, los sitios de los que provienen los conjuntos líticos. (Fuente: Modificado a partir de imagen satelital de Google Earth Pro). Derecha: Fotografías generales de tres de los sitios arqueológicos abordados (Fuente: Parque de Las Leyendas, 2014).

Figure 3: Left: Map of the location of the nine selected sites. In white line spacing, the perimeter of the CAM in the PATPAL. In yellow, the sites where lithic assemblages come from. (Source: Modified from satellite image of Google Earth Pro). Right: General photographs of three of the archaeological sites addressed (Source: Parque de Las Leyendas, 2014).

esta técnica se utilizó para caracterizar de forma semicuantitativa a las andesitas sobre guijarro. Para la DRX, se siguieron las siguientes etapas: preparación, irradiación y análisis de las muestras. Las muestras pulverizadas fueron llevadas al Laboratorio de Rayos X del INGEMMET, donde fueron colocadas en láminas a presión, para luego ser irradiadas. Se utilizó el equipo X-Ray Diffractometer Lab X Shimadzu XRD-6000. El análisis de la muestra tuvo por objetivo identificar el tipo de roca a partir del espectro obtenido anteriormente. Se utilizó la base de datos del INGEMMET para proceder a la identificación de los minerales.

Tabla 1. Conjuntos líticos estudiados en el análisis tecnológico y asociación cronológica relativa

Table 1: Lithic assemblages studied in technological analysis and relative chronological association

\begin{tabular}{|c|c|c|c|c|c|c|c|c|c|c|c|c|}
\hline \multirow{4}{*}{$\mathbf{N}^{\circ}$} & \multirow{4}{*}{ Sitio } & \multirow{4}{*}{$\begin{array}{l}\text { Cronología } \\
\text { relativa }\end{array}$} & \multirow{4}{*}{$\begin{array}{c}\text { Período } \\
\text { (sensu Rowe 1962) }\end{array}$} & \multicolumn{8}{|c|}{$\mathrm{N}^{\circ}$ de artefactos } & \multirow{4}{*}{$\begin{array}{l}\text { Total de artefactos } \\
\text { por conjunto lítico }\end{array}$} \\
\hline & & & & \multirow{2}{*}{\multicolumn{2}{|c|}{ Núcleos }} & \multicolumn{4}{|c|}{ Tallados } & \multirow{3}{*}{$\begin{array}{c}\text { Total de artefactos } \\
\text { tallados }\end{array}$} & \multirow{3}{*}{$\begin{array}{l}\text { Macro- } \\
\text { utillaje }\end{array}$} & \\
\hline & & & & & & \multicolumn{2}{|c|}{ Lascas } & \multicolumn{2}{|c|}{ Útiles } & & & \\
\hline & & & & $\mathrm{n}$ & $\%$ & $\mathrm{n}$ & $\%$ & $\mathrm{n}$ & $\%$ & & & \\
\hline 1 & $\begin{array}{l}\text { Huaca El } \\
\text { Rosal }\end{array}$ & $\sim 1800-1400 \mathrm{BP}$ & Período Intermedio Temprano & 85 & 24.29 & 6 & 2.30 & 79 & 15.90 & 170 & 21 & 191 \\
\hline 2 & $\begin{array}{c}\text { Huaca } \\
\text { Middendorf }\end{array}$ & $\sim 1800-1250 \mathrm{BP}$ & $\begin{array}{l}\text { Periodo Intermedio Temprano- } \\
\text { inicios Horizonte Medio }\end{array}$ & 8 & 2.29 & 7 & 2.68 & 18 & 3.62 & 33 & 6 & 39 \\
\hline 3 & Huaca 33 & $\sim 1800-1400 \mathrm{BP}$ & Periodo Intermedio Temprano & 73 & 20.86 & 53 & 20.31 & 22 & 4.43 & 148 & 33 & 181 \\
\hline 4 & Huaca 58B & $\sim 1800-1400 \mathrm{BP}$ & Periodo Intermedio Temprano & 43 & 12.29 & 25 & 9.58 & 102 & 20.52 & 170 & 3 & 173 \\
\hline 5 & Huaca 34 & $\sim 1050-470 \mathrm{BP}$ & Período Intermedio Tardío & 26 & 7.43 & 86 & 32.95 & 114 & 22.94 & 226 & 12 & 238 \\
\hline 6 & Huaca 43 & $\sim 1050-470 \mathrm{BP}$ & Periodo Intermedio Tardio & 20 & 5.71 & 16 & 6.13 & 13 & 2.62 & 49 & 4 & 53 \\
\hline 7 & Muralla 46A & $\sim 1050-470 \mathrm{BP}$ & Periodo Intermedio Tardío & 14 & 4.00 & 8 & 3.07 & 10 & 2.01 & 32 & 2 & 34 \\
\hline 8 & Muralla 55E & $\sim 470-400 \mathrm{BP}$ & Horizonte Tardio & 8 & 2.29 & 5 & 1.92 & 14 & 2.82 & 27 & 1 & 28 \\
\hline 9 & $\begin{array}{l}\text { Huaca San } \\
\text { Miguel }\end{array}$ & $\sim 470-400 \mathrm{BP}$ & Horizonte Tardio & 73 & 20.86 & 55 & 21.07 & 125 & 25.15 & 253 & 67 & 320 \\
\hline \multicolumn{4}{|c|}{ Total } & 350 & 100.00 & 261 & 100.00 & 497 & 100.00 & 1108 & 149 & 1257 \\
\hline
\end{tabular}


Debido a que sólo tratamos aquí de la génesis instrumental, de la totalidad de artefactos tallados $(n=1108)$, presentamos aquí la información concerniente a los útiles identificados ( $n=497)$, que fueron objeto de análisis tecno-funcional (sensu Boëda, 2013). Este análisis permitió reconocer los criterios técnicos específicos de cada útil, lo que condujo al establecimiento hipotético de las Unidades Tecno-Funcionales (UTF) transformativa (UTFt) y prensil (UTFp). Una Unidad Tecno-Funcional se refiere a un conjunto de elementos y/o caracteres técnicos que coexisten en una sinergia de efectos (Boëda, 1997, p. 34. La traducción es nuestra). Las UTF pueden participar en la constitución de una zona transformativa (en contacto con la materia a transformar), prensil (zona en contacto con el operador) y transmisora (zona que recibe la energía emitida por el operador y la transmite a la zona transformativa) (Boëda, 2013, p. 51). Sin embargo, en el caso de las colecciones aquí estudiadas, la zona transmisora de la energía generalmente está incluida en la UTFp.

Las UTF fueron reagrupadas en tecno-tipos, noción que se refiere a la asociación entre la configuración volumétrica resultante de las modalidades de producción (es decir, los soportes) y la organización funcional de los pares de UTF(t)/UTF(p) (Boëda, 1997).

Luego de un muestreo basado en la calidad de los contextos arqueológicos, los tecno-tipos determinados fueron analizados a nivel de microvestigios vegetales (Babot, Lund, \& Olmos, 2016), a fin de obtener información directa sobre los usos probables. El análisis de microvestigios se centró, especialmente, en los granos de almidón (GA), sin embargo también se consideraron, en términos de presencia y ausencia, a los silicofitolitos (Sif), fitolitos de calcio (FCa), anillos de celulosa $(A C)$, microcarbones (Mic), diatomeas (Di), esferulitas (Esf) y tejidos silicificados (Te) (Babot, 2009). Una muestra de 39 artefactos fue conformada a partir de los siguientes criterios: la presencia efectiva de cada tecno-tipo en un sitio arqueológico y la calidad de su contexto de procedencia, a fin de evitar posibles casos de contaminación (Tabla 2).

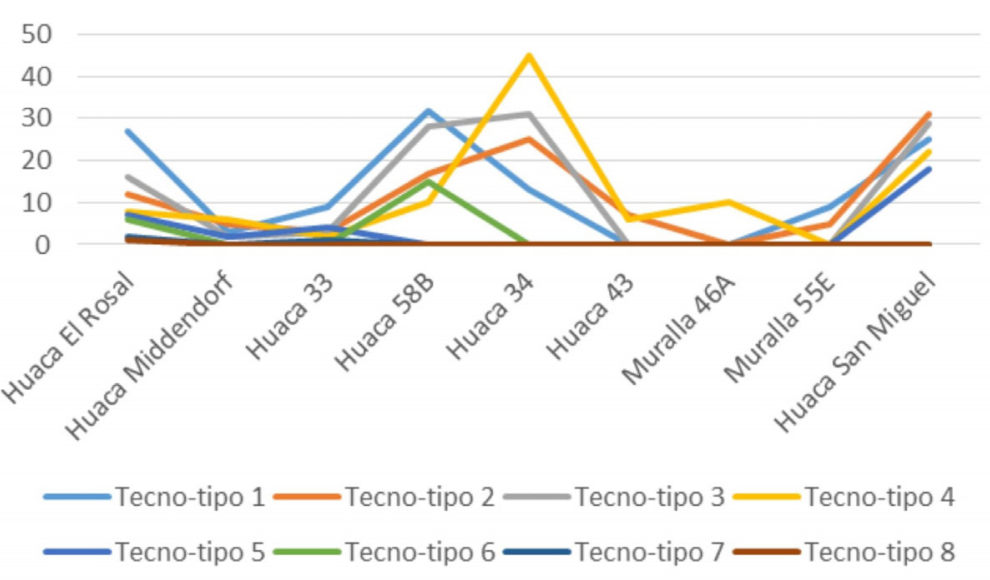

Para el caso de nuestra muestra artefactual, se diseñó un " protocolo de bajo impacto » (Babot et al., 2016), que consistió en una intervención mínima durante el proceso de extracción de los microvestigios. Este protocolo se compuso de dos grandes etapas: (1) confección de un herbario y colección de referencia histológica a partir de material vegetal moderno y (2) recuperación, análisis e identificación de los microvestigios preservados en las UTFt de los tecno-tipos establecidos. Esta etapa se realizó íntegramente en el Laboratorio de Arqueobotánica del Instituto de Arqueología y Museo, Universidad Nacional de Tucumán (IAM, UNT). Se procedió al muestreo de los útiles correspondientes a los ocho tecno-tipos previamente establecidos. Se ubicaron las UTFt, y dentro de ellas, los sectores con residuos visibles, o bien las irregularidades de la superficie como poros, vesículas y grietas. La recuperación de los residuos se realizó a partir de dos técnicas, muestreo en seco estratificado y estandarizado por área, y muestreo en húmedo por goteo utilizando una alícuota constante (Babot 2009). Antes de cada extracción, se realizó la limpieza mecánica por cepillado suave de la superficie de cada artefacto, con el objetivo de obtener una muestra de control relacionada a la naturaleza de los microvestigios propios de la matriz sedimentaria del contexto de deposición del artefacto. Tras la recuperación y montaje de las muestras, se procedió con la observación microscópica. Los microvestigios fueron observados a medianos aumentos (100-400x), en un microscopio petrográfico Leica DM750 P con kit de polarización y cámara digital incorporada. Las imágenes obtenidas fueron procesadas a través del software LAS EZ (Leica Application Suite). Cada muestra escaneó de forma completa a través de transectas paralelas horizontales, barriendo la totalidad de la superficie $(24 \times 24 \mathrm{~mm})$.

Finalizado el análisis, se procedió a la asignación taxonómica y anatómica de los microvestigios arqueológicos, a través de la comparación con la colección de referencia elaborada previamente y con material édito en el caso de tazones no incluidos en ella, y ya identificados por otros autores.

Al término de las dos etapas mencionadas anteriormente, se procedió a la evaluación de la presencia de los taxones identificados, en función de cada período arqueológico y en relación con la variabilidad instrumental (tecno-tipos). Para ello, se utilizó el concepto de ubicuidad o análisis de presencia (sensu Popper, 1988), que permite determinar el número de veces

Figura 4. Frecuencia de tecno-tipos por sitio arqueológico

Figure 4. Frequency of techno-types by archaeological site 


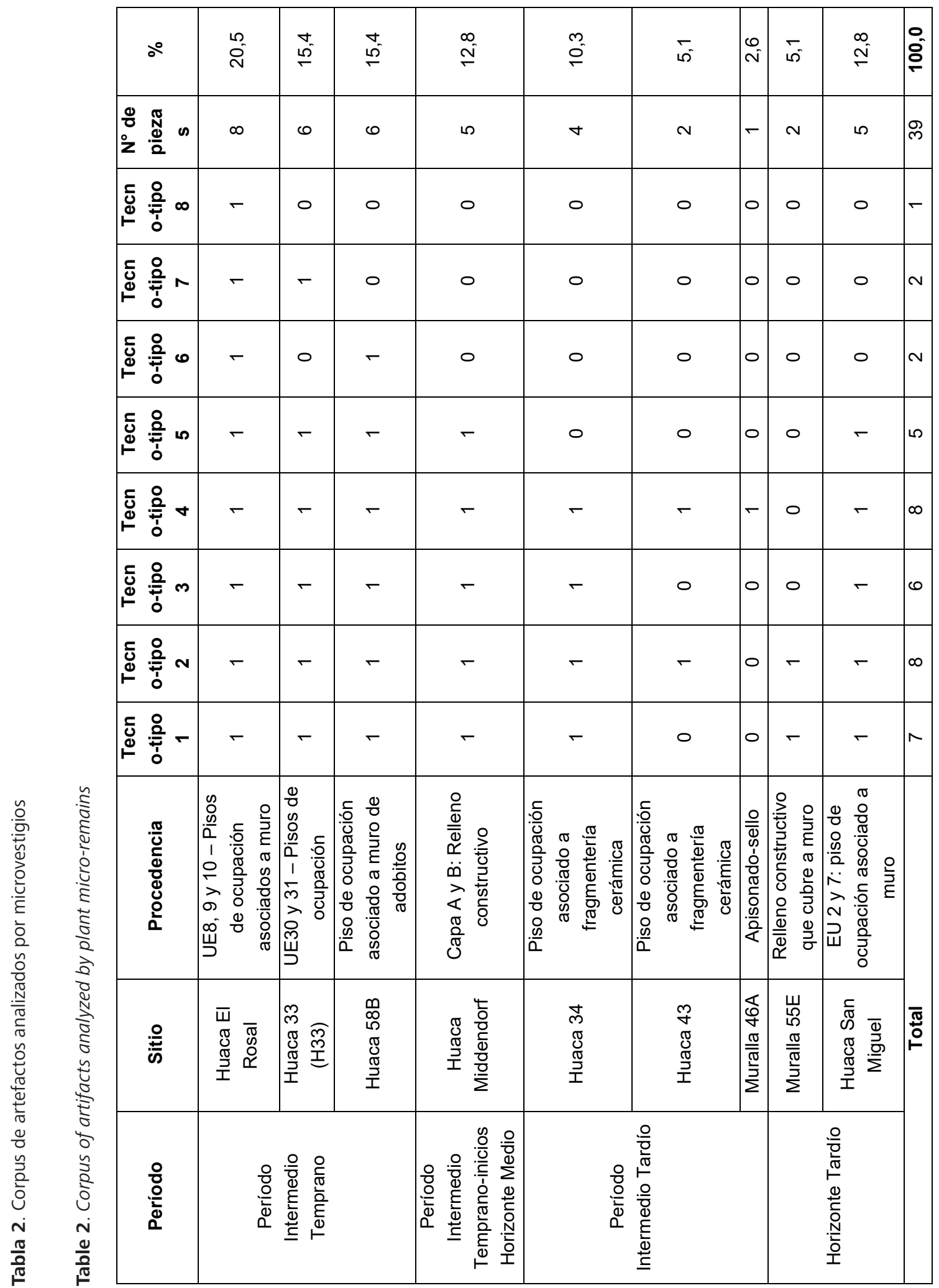

que un taxón aparece en un conjunto de muestras.

\section{Resultados}

Previamente, el análisis tecnológico de la producción permitió identificar un predominante aprovisionamiento local de diferentes rocas ígneas, así como tres sistemas de débitage presentes en todos los períodos, pero no en todos los sitios: unipolar (C1), alternante (D1) y bipolar (F3) (Pérez, 2018).

\section{Petrografía}

El análisis petrográfico a nivel macro permitió identificar la selección de guijarros y rodados de roca ígnea volcánica 
Tabla 3: Descripción de tecno-tipos en el CAM-PATPAL

Table 3: Description of techno-types in CAM-PATPAL.

como materia prima exclusiva para la manufactura del utillaje en todos los conjuntos. Esta roca se presenta compacta, con altas densidades, dureza media, sin mayores fracturas abiertas ni presencia de fragmentos líticos, y en varios colores (grises con diferentes tonalidades); mientras que sus texturas pueden variar entre afaníticas, faneríticas y porfiríticas, siendo predominantes las primeras $(n=320,64.4 \%)$. Por su parte, el análisis microscópico de sección delgada permitió afinar la clasificación macroscópica y determinar una explotación predominante de andesitas afaníticas. Se trata, generalmente, de una textura conformada por cristales de plagioclasas alterados, además de moldes de cristales inmersos en una matriz microcristalina, constituida por plagioclasas y agregados ferromagnesianos, reemplazos por epidota y feldespatos potásicos.

\section{Difracción de Rayos $X(D R X)$}

Se pudo determinar una mineralogía muy específica para el grupo de las andesitas microporfídicas: principalmente cuarzo (entre $40 \mathrm{y}$ $46 \%$ ), anortita (entre 40 y $80 \%$ ), albita (40\%) y magnetita (11\%). El dato más resaltante constituye el porcentaje de $\mathrm{SiO}_{2}$, que en todas las muestras alcanza un porcentaje de alrededor del 40\%. Este porcentaje excede los valores promedio de las andesitas, sin embargo, es coherente con la sílice secundaria que compone los demás minerales.

\section{Análisis tecno-funcional}

Previo al façonnage, se seleccionaron soportes (blanks) de guijarros de sección elipsoide, generalmente entre 60 y $80 \mathrm{~mm}$ de largo, 60 y $90 \mathrm{~mm}$ de ancho y entre 30 y $50 \mathrm{~mm}$ de espesor. De forma general, los esquemas de façonnage implican dos o tres levantamientos parciales en ambas o una sola cara del guijarro, a fin de configurar un diedro cortante asimétrico. La etapa de confección corresponde al retoque unifacial de estos diedros, formando principalmente ángulos abruptos

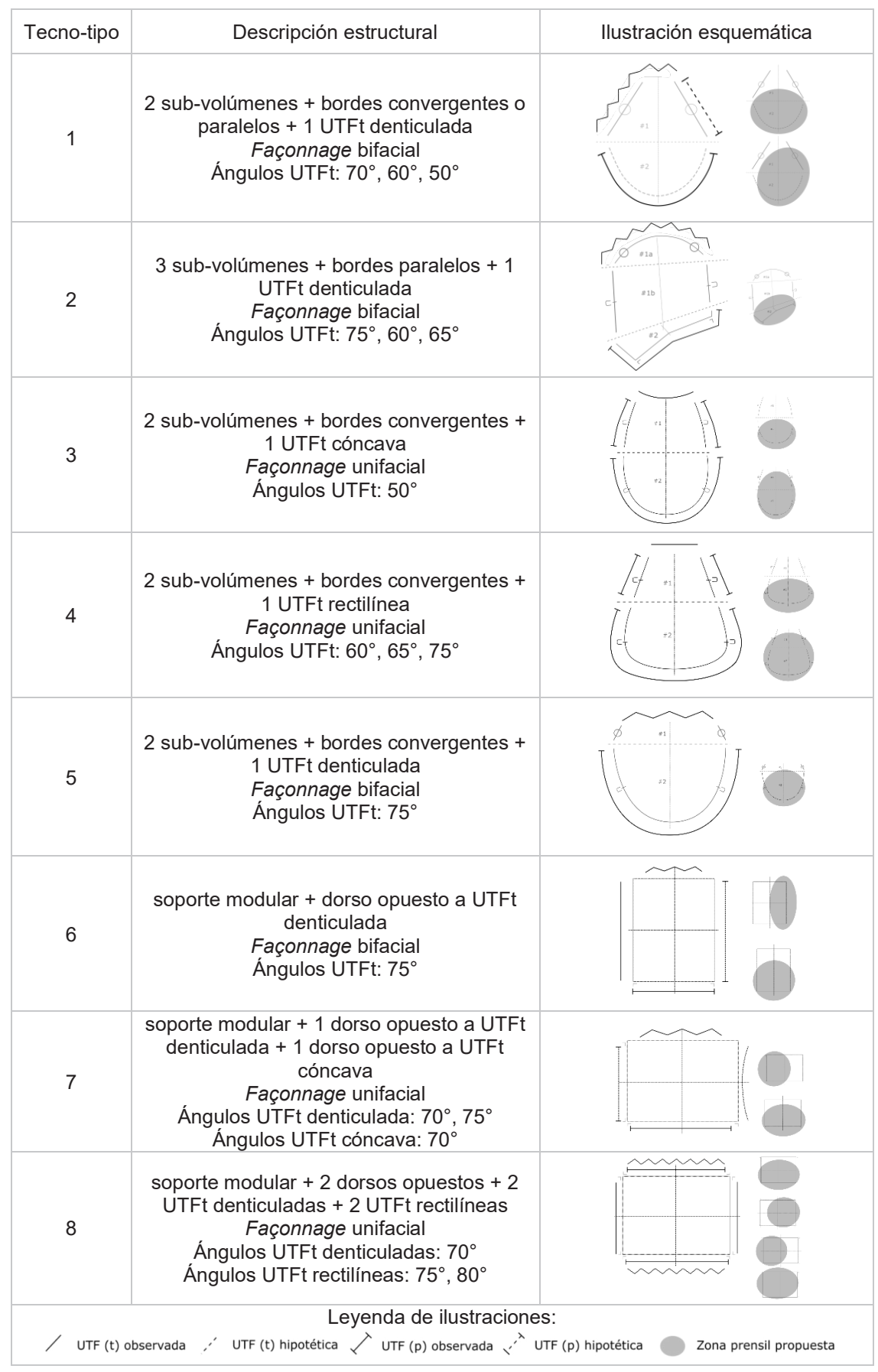

(entre $70^{\circ}$ y $\left.80^{\circ}\right)$

En todos los útiles analizados, se identificaron 528 UTFt, con delineación denticulada, convexa, cóncava y rectilínea, siendo predominante la primera $(n=291$, $58.55 \%)$. Las UTFp identificadas ( $n=502)$ corresponden en su gran mayoría a la parte cortical de los guijarros seleccionados, en posición opuesta a las UTFt, de sección elipsoide, compuestas por dos bordes redondeados con diferentes grados de divergencia entre sí. En algunos casos ( $n=102,20.31 \%$ ), las UTFp fueron objeto de configuración, siempre a través de cortos levantamientos por façonnage unifacial. Al agrupar las UTFt y UTFp observadas, se determinaron ocho tecno-tipos (T-t) 
(Tabla 3), de los cuales los seis primeros cuentan con una sola UTFt, mientras que los dos últimos (tecno-tipos 7 y 8), cuentan con dos o más UTFt. Ahora bien, si nos detenemos en la frecuencia de los tecno-tipos por sitio arqueológico, es evidente la predominancia de los tecnotipos 1 al 4 (88\% del total de útiles), mientras que los tecno-tipos 5 al 8 se presentan en baja proporción (12\%) (Figura 4) Además, ningún tecno-tipo está presente en todos los sitios, aunque el tecno-tipo 4 ocurre en todos los sitios, con la excepción de la Muralla 55E.

\section{Microvestigios vegetales}

En todos los 39 artefactos analizados, fue posible identificar microvestigios vegetales, entre ellos: 6 morfotipos de granos de almidón, 2 morfotipos de silicofitolitos y 1 relativo a tejidos vegetales desecados. Los morfotipos de granos de almidón identificados corresponden a Caesalpinia spinosa, Prosopis, Zea mays, Stipa, Cucurbita ficifolia y Solanum tuberosum (Tabla 4) (Pérez, 2018).

De forma general, puede señalarse que los útiles de Huaca El Rosal, Huaca Middendorf, Huaca 33 y Huaca San Miguel presentan mayor frecuencia y variabilidad de microvestigios, mientras que los correspondientes a la Muralla 46A, Muralla 55E y Huaca 43 constituyen el caso contrario. Finalmente, del conjunto de recursos vegetales identificados, Zea mays ("maíz") y Caesalpinia spinosa ("tara") representan el 43\% y 33\%, respectivamente, superando ampliamente la frecuencia alcanzada por los otros recursos (Prosopis, 7\%; Stipa, 5\%, Cucurbita ficifolia, 8\%; Solanum tuberosum, 4\%). Aunque recurrentes de forma diferencial de acuerdo al período arqueológico, ambos recursos predominan en todo el instrumental analizado (Tabla 4) Un ejemplo de tecnotipo y de micro-vestigios recuperados se muestra en la Figura 5.

\section{Discusión y conclusiones preliminares}

Los procesos de instrumentalización (Artesano --> Artefacto)

A nivel de los constreñimientos propios de la materia prima, el análisis petrográfico macro y microscópico permitió indagar en los aspectos más cualitativos ligados al aprovisionamiento y gestión de guijarros de roca ígnea volcánica. Por su parte, la DRX ofreció la oportunidad de profundizar en los aspectos cuantitativos de la matriz microcristalina y corroborar la clasificación de andesitas microporfídicas. A su vez, la definición de estas características permite señalar que la alta densidad y el alto contenido de sílice en los rodados disponibles localmente son los criterios principales que explican su selección para la producción de filos altamente resistentes. Por otro lado, la morfología y sección elipsoide de los guijarros iniciales también constituye un criterio técnico potencial para la obtención de los funcionamientos requeridos. Por esta razón, los tecno-tipos identificados son el reflejo de la fuerte selección realizada antes de la confección. En un sentido ergonómico, el artefacto posteriormente producido se encuentra potencialmente organizado en los guijarros y rodados iniciales, por lo que una larga etapa de confección no resultó necesaria.

\section{Los procesos de instrumentación (Artefacto -->} Artesano)

Ahora bien, una variabilidad tecno-funcional importante se expresa en la presencia de ocho tecno-tipos, manifestados efectivamente en 39 artefactos analizados, que pueden ser adscritos a la transformación de recursos vegetales específicos. A este respecto, la integración del análisis tecno-funcional y de microvestigios (Tabla 5), permitió determinar que durante el Período Intermedio Temprano e inicios del Horizonte Medio se utilizaron siete tecno-tipos para un solo recurso, Caesalpinia spinosa ("tara"): tecno-tipos 1, 2, 3, 5, 6, 7 y 8. En el Período Intermedio Tardío, se sigue utilizando el tecno-tipo 1, pero además se emplea uno nuevo, el tecnotipo 4. En el Horizonte Tardío, únicamente los tecno-tipos 2, 5, 6,7 y 8 son utilizados

\begin{tabular}{|c|c|c|c|c|c|c|c|c|c|c|c|c|}
\hline \multirow{3}{*}{ Poales } & \multirow{3}{*}{ Poaceae } & & & & & & & & & & & \\
\hline & & Zea mays L. & Maíz & 28 & 58 & 0 & 9 & 0 & 0 & 0 & 0 & 95 \\
\hline & & Stipa L. & - & 6 & 6 & 0 & 0 & 0 & 0 & 0 & 0 & 12 \\
\hline $\begin{array}{c}\text { Cucurbitale } \\
\qquad \mathrm{s}\end{array}$ & Cucurbitáceae & $\begin{array}{c}\text { Cucurbita ficifolia } \\
\text { Bouché }\end{array}$ & $\begin{array}{l}\text { Calabaza } \\
\text { blanca, } \\
\text { chiclayo, } \\
\text { lacayote }\end{array}$ & 13 & 5 & 0 & 0 & 0 & 0 & 0 & 0 & 18 \\
\hline Solanales & Solanaceae & $\begin{array}{c}\text { Solanum tuberosum } \\
\text { L. }\end{array}$ & Papa & 10 & 0 & 0 & 0 & 0 & 0 & 0 & 0 & 10 \\
\hline & N.I. ( & identificados) & & 32 & 71 & 10 & 84 & 17 & 16 & 20 & $\begin{array}{l}1 \\
8\end{array}$ & 268 \\
\hline & & TOTAL & & 177 & $\begin{array}{l}14 \\
0\end{array}$ & 10 & 93 & 17 & 16 & 20 & $\begin{array}{l}1 \\
8\end{array}$ & 491 \\
\hline
\end{tabular}


Tabla 5. Presencia de cada taxón según el período arqueológico, el sitio y el tecno-tipo en el CAM-PATPAL

Table 5. Presence of each taxon according to the archaeological period, the site and the techno-type in the CAM-PATPAL

\begin{tabular}{|c|c|c|c|c|c|}
\hline $\begin{array}{l}\frac{0}{\circ} \\
\frac{0}{0} \\
\frac{0}{0}\end{array}$ & $\begin{array}{l}\text { Tecno- } \\
\text { tipo } \\
\text { Sitio }\end{array}$ & 1 & $2,5,6,7,8$ & 3,8 & 4 \\
\hline \multirow{4}{*}{ 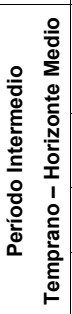 } & Huaca EI Rosal & $\begin{array}{c}\text { Caesalpinia spinosa } \\
\text { Zea mays }\end{array}$ & $\begin{array}{c}\text { Solanum tuberosum } \\
\text { Caesalpinia spinosa } \\
\text { Zea mays }\end{array}$ & Cucurbita ficifolia & $\begin{array}{c}\text { Zea mays } \\
\text { Cucurbita ficifolia }\end{array}$ \\
\hline & $\begin{array}{c}\text { Huaca } \\
\text { Middendorf }\end{array}$ & Caesalpinia spinosa & Caesalpinia spinosa & Caesalpinia spinosa & $\begin{array}{c}\text { Zea mays } \\
\text { Cucurbita ficifolia }\end{array}$ \\
\hline & Huaca 58B & Caesalpinia spinosa & Caesalpinia spinosa & Caesalpinia spinosa & \\
\hline & Huaca 33 & & Caesalpinia spinosa & Caesalpinia spinosa & \\
\hline \multirow{3}{*}{ 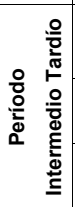 } & Huaca 34 & $\begin{array}{c}\text { Caesalpinia spinosa } \\
\text { Stipa sp. }{ }^{*}\end{array}$ & & Prosopis sp. & \\
\hline & Huaca 43 & & & & Caesalpinia spinosa \\
\hline & Muralla 46A & & & & Caesalpinia spinosa \\
\hline \multirow{2}{*}{ 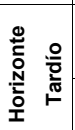 } & $\begin{array}{l}\text { Huaca San } \\
\text { Miguel }\end{array}$ & & $\begin{array}{c}\text { Caesalpinia spinosa } \\
\text { Stipa sp. }{ }^{*}\end{array}$ & & \\
\hline & Muralla 55E & Zea mays & Zea mays & & \\
\hline
\end{tabular}

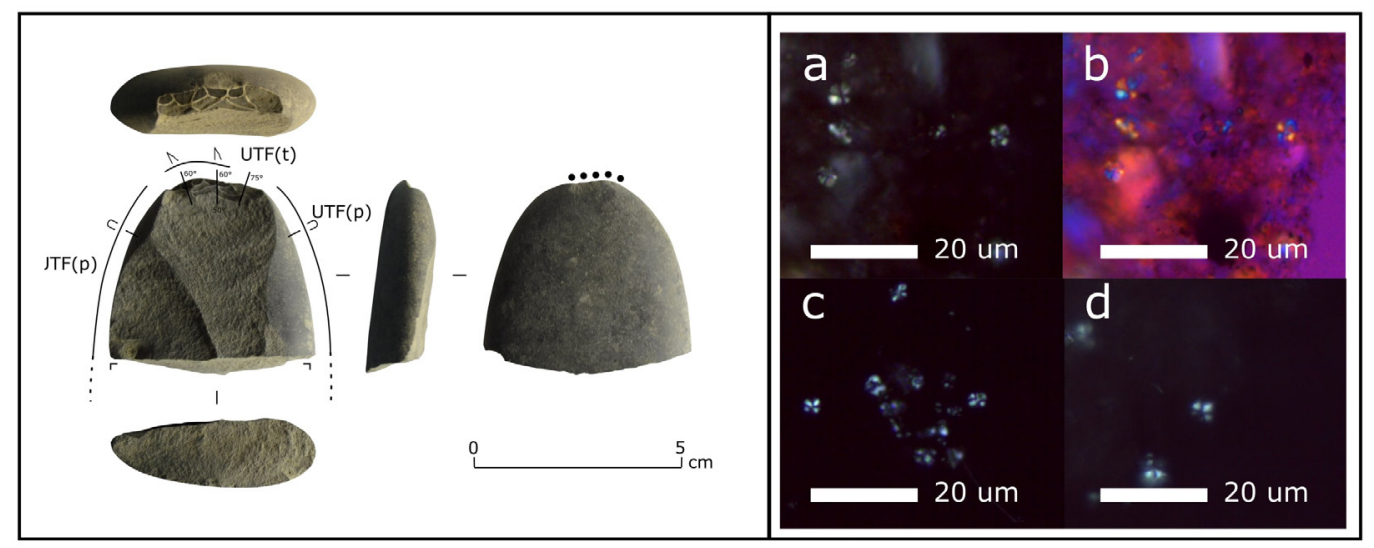

Figura 5. Huaca 43, Tecno-tipo 4, (a y b) granos de almidón recuperados en la única UTF (t), asignación taxonómica: Caesalpinia spinosa («tara»). (c y d) Granos de almidón modernos de la misma especie.

Figure 5. Huaca 43, Techno-type 4, ( $a$ and b) starch grains recovered in the single UTF (t), taxonomic assignment: Caesalpinia spinosa ("tara"). (c and d) Modern starch grains of the same species.

para procesar este recurso vegetal. En lo que concierne a otros recursos, la recurrencia es mínima. Evidentemente, las tendencias mencionadas están estrechamente relacionadas con la función de cada sitio y el contexto arqueológico de procedencia, hecho que se evidencia en la baja frecuencia de estos recursos vegetales y tecnotipos en las Murallas 46A y 55E, estructuras atribuidas a funciones defensivas.

Desde la evaluación global de los resultados esbozados es posible señalar que la muestra analizadas procedente del CAM-PATPAL es la expresión de una selección minuciosa de los volúmenes naturales disponibles localmente, una configuración volumétrica y funcional determinada por esta selección y específica al procesamiento de determinados recursos vegetales. Esta expresión instrumental cambia a través del tiempo, produciéndose una suerte de estandarización hacia el Horizonte Tardío.

Finalmente, y a pesar de no cubrir con todo el espectro de elementos que involucran los procesos de instrumentalización e instrumentación, este abordaje ergonómico se muestra como un útil teórico-metodológico capaz de integrar de forma transversal un conjunto de técnicas especializadas y orientarlas a una concepción diferente (netamente funcional) del utillaje lítico y la actividad artesanal. Esta concepción diferente se presenta 
esencialmente diacrónica, acoplando el análisis tecnofuncional al análisis de microvestigios.

\section{Agradecimientos}

Nuestro agradecimiento profundo a Daniel Campi, Carlos Aschero, Salomón Hocsman, Julieta Zapatiel, Mónica Burgos y Lorena Cohen por su apoyo en el Laboratorio de Arqueobotánica del Instituto Superior de Estudios Sociales (ISES-CONICET/UNT). También expresamos nuestro agradecimiento a Gissella Tuesta y Nadiezhda Alva por su colaboración en el Gabinete de Arqueología del Parque de las Leyendas "Felipe Benavides Barreda". Finalmente, vaya nuestro agradecimiento a los ingenieros Oscar Bernuy, Edwin Loayza y Miguel Chumbe por su colaboración en el análisis petromineralógico llevado a cabo en el Instituto Geológico Minero y Metalúrgico del Perú (INGEMMET). Finalmente, nuestro agradecimiento a Santiago Uceda y Eric Boëda, quienes ofrecieron el soporte necesario para llevar a cabo esta investigación. Este trabajo fue financiado por una Beca CONCYTEC 2016-2018.

\section{Bibliografía}

Babot, P. (2009). La cocina, el taller y el ritual: explorando las trayectorias del procesamiento vegetal en el noroeste argentino. Darwiniana 47, 1, 7-30.

Babot, P., Lund, J., \& Olmos, V. (2016). Tres técnicas de muestreo complementarias en colecciones de referencia de micropartículas. Experiencias de su aplicación conjunta. En A. Zucol, N. Patterer, M. Colobig, \& E. Moya (Eds.), II Taller "Micro Paleoetnobotánica: relevancia de una red interdisciplinaria de investigadores en fitolitos y almidones". Libro de Resúmenes (pp. 12-17). Diamante.

Boëda, E. (1991). Approche de la variabilité des systèmes de production lithique des industries du Paléolithique inférieur et moyen: chronique d'une variabilité attendue. Techniques et culture, 17-18, 37-79.

Boëda, E. (1997). Technogénèse des systèmes de production lithique au Paléolithique moyen inférieur et moyen en Europe occidentale et au Proche-Orient (Habilitation à diriger des recherches, 2 vol.). Université de Paris X - Nanterre, Nanterre.

Boëda, E. (2013). Techno-logique \& Technologie. Une Paléohistoire des objets lithiques tranchants. Prigonrieux: @rchéoéditions.com.

Canziani, J. (2009). Ciudad y territorio en los Andes: contribuciones a la historia del urbanismo prehispánico. Lima: Fondo Editorial de la Ponti-ficia Universidad Católica del Perú.

Carrión, L., \& Narváez, J. (2014). Descripción general de Maranga. En L. Carrión \& J. Narváez (Eds.), Arqueología. Catorce Años de Investigaciones en Maranga (pp. 33-52). Lima: Municipalidad de Lima Metropolitana y Patronato del Parque de las Leyendas-Felipe Benavides Barreda.

Díaz, L., \& Vallejo, F. (2004). Variaciones culturales en el valle de Lima durante la ocupación incaica. Chungará, Revista de Antropología Chilena, 36, 295-302.

Kaulicke, P. (2000). La Sombra de Pachacamac: Huari en la Costa Central. Boletín de Arqueología PUCP, 4, 313-358.
Lepot, M. (1993). Approche techno fonctionnelle de l'outillage lithique moustérien : essai de classification des parties actives en termes d'efficacité technique. Application à la couche M2e sagittale du Grand Abri de La Ferrassie (Fouille Henri Delporte). (Tesis de maestría). Université Paris X - Nanterre, Nanterre.

Leroi-Gourhan, A. (1964). Le geste et la parole Vol. I: Technique et langage. Paris: Albin Michel.

Makowski, K. (2001). Las civilizaciones prehispánicas en la Costa Central y Sur. En Historia de la cultura peruana: Vol. I (pp. 163243). Lima: Fondo Editorial del Congreso del Perú.

Makowski, K. (2002). Power and Social Ranking at the End of the Formative Period. The Lower Lurin Valley Cemeteries. En W. Isbell \& H. Silverman (Eds.), Andean Archaeology I. Variations in Sociopolitical Organization (pp. 89-120). New York: Kluwer Academic/Plenum Publishers.

Marcone, G. (2015). Proceso político y reorganización social lima desde la perspectiva de los grupos intermedios: el ejemplo del Lote B. Boletín de Arqueología PUCP, 19, 171-190.

Mauricio, A., Prieto, G., \& Pardo, C. (2014). Avances en la arqueología de la cultura Lima. Boletín de Arqueología PUCP, $18,5-14$.

Narváez, J. (2013). Precolonial irrigation and settlement patterns in three arti-cial valleys in Lima, Peru (Tesis de doctorado). University of Calgary, Calgary.

Parque de Las Leyendas (Ed.). (2014). Cuaderno de investigaciones de la división de arqueología Nro. 2. Lima: Parque de las Leyendas, Hynoscha Import SAC.

Pérez, A. (2018). Étude fonctionnelle des artefacts lithiques tardifs ( 200 av. J.C.-1532 apr. J.-C.) du Complexe Archéologique Maranga (Côte Centrale, Pérou) (Tesis de maestría). Université de Rennes 1 , Rennes.

Popper, V. (1988). Selecting quantitative measurements in paleoethnobotany. En C. Hastorf \& V. Popper (Eds.), Current Paleoethnobotany. Analytical methods and cultural interpretations of archaeological plant remains (pp. 53-71). Chicago: University of Chicago Press.

Rabardel, P. (1995). Les Hommes et les technologies. Aproche cognitive des instruments contemporains. Paris: Armand Colin.

Silva, E. (2014). Los artefactos líticos del Parque de las Leyendas: del Horizonte Medio al Horizonte Tardío. En L. Carrión \& J. Narváez (Eds.), Arqueología. Catorce años de investigaciones en Maranga (pp. 186-209). Lima: Municipalidad de Lima Metropolitana y Patronato del Parque de las Leyendas-Felipe Benavides Barreda.

Taschini, M. (1968). L'industrialitica Pre-incaica di Cajamarquilla (Peru). Bulletino di Paletnologia Italiana, Nuova serie XIX, 185225.

Tixier, J., Inizan, M.-L., \& Roche, H. (1980). Préhistoire de la Pierre taillée Tome I. Terminologie et technologie. Valbonne: Cercle de recherches et d'études préhistoriques.

Trouche, L. (2005). An instrumental approach to mathematics learning in symbolic calculator environments. En D. Guin, K. Ruthven, \& L. Trouche (Eds.), The Didactical Challenge of Symbolic Calculators. Turning a Computational Device into a Mathematical Instrument (pp. 137-162). USA: Springer. 